\title{
Menstrual Hygiene Among Medical Female Students
}

\section{Mihail Kochubovski ${ }^{1,2}$, Aleksandra Petrova ${ }^{1,2 *}$, Shaban Memeti ${ }^{1,2}$ and Djansun Bukovec ${ }^{2}$}

${ }^{1}$ Institute of Public Health, Skopje, Republic of North Macedonia

${ }^{2}$ University Ss. Cyril and Methodius, Medical Faculty, Skopje, Republic of North

Macedonia

*Corresponding Author: Aleksandra Petrova, MD Researcher, Department of Water

Safety and Environmental Sanitation, Skopje, Republic of North Macedonia.
Received: June 02, 2021

Published: September 20, 2021

(C) All rights are reserved by Aleksandra

Petrova.

\section{Abstract}

Menstruation, or period, is a monthly occurrence for people of reproductive age. The number of girls, women, transgender men and non-binary persons that experience the period is about 1.8 billion. Even though a big progress is made in raising the awareness for menstruation hygiene and health, however, there are some issues that cause girls and women not to meet the basic menstruation hygiene and health needs. Researchers say that poverty, menstruation taboos, lack of basic hygiene services, gender inequality have negative impact on the lives of the vulnerable categories. Adolescent girls during their period, are often faced with intimidation, stigma, and even social discrimination. The aim of the work is to analyze the habits during the menstrual cycle as well as the absence from classes. The study is a cross sectional observational study conducted between November and December 2019. The sampling

\section{Introduction}

Menstruation, or period, is a monthly occurrence for people of reproductive age. The number of girls, women, transgender men and non-binary persons that experience the period is about 1.8 billion. Millions among these people are not able to provide appropriate health and hygiene care during their monthly period. Even though a big progress is made in raising the awareness for menstruation hygiene and health. Researchers say that poverty, menstruation taboos, lack of basic hygiene services, gender inequality have negative impact on the lives of the vulnerable categories. Adolescent girls during their period, are often faced with intimidation, stigma, and even social discrimination. Beside adolescent girls that face problems during their period, transgender men and non-binary persons are constantly deprived to perform their basic needs. All of these issues result negatively on the every-day activities of those who menstruate. It usually affects their mobility, social life, school attending, even causing stress and anxiety disorder. These problems are usually met among girls and women in humanitarian crisis. Access to safe drinking water and sanitation is a human right that is part of the right to adequate standard of living, enshrined in article 11 of the International Covenant on Economic, Social and Cultural Rights. It has been explicitly recognized by the General Assembly of the United Nations and by the United Nations Human Rights Council. To comply with this obligation, states are legally obliged to provide access to water and sanitation for all members of the population and must take steps towards the full realization of the right. Availability, safety and unlimited access to water and sanitation is a human right. Under the Water and Health Protocol, parties must provide access to water and sanitation for all. This documented Protocol actually binds the parties to equitable access, especially for those members of the population who are socially disadvantaged, through specific targets tailored to each country accordingly [1]. The beginning of menstruation for some girls can be a vulnerable period. In adolescence, Mmenstrual health and hygiene 
(MHH) are the starting point for sexual and reproductive health education. Strengthening Menstrual Health and Hygiene Programs can help girls build skills overcoming obstacles, feeling free, and self-confident. MHH (menstrual health and hygiene) is important for the fulfilment of girls' and women's rights, a key objective of the Sustainable Development Goals (SDGs). Women and girls' access to MHH is a component of gender-responsive WASH services; SDG 6.2 acknowledges the right to menstrual health and hygiene, with the explicit aim to, "By 2030, achieve access to adequate and equitable sanitation and hygiene for all and end open defecation, paying special attention to the needs of women and girls and those in vulnerable situations". Without considering needs for safe and dignified menstruation, the world cannot achieve the vision for sanitation and hygiene under Goal 6 [2]. The aim of the work is to analyse the habits during the menstrual cycle as well as the absence from classes.

\section{Materials and Methods}

The study is a cross sectional observational study conducted between November and December 2019. The sampling unit includes the Medical Faculty in Skopje, in North Macedonia. The subjects included were adolescent school girls aged 22-25 years of grades $\mathrm{V}$ who had attained menarche and had consented to participate. All the students in classes were included in the study. The questionnaire was used to determine the level of menstrual hygiene practices, Menstrual Hygiene in Medical Students», which consisted of 34 questions (most of which are multiple choice), adapted from of Questionnaire Assessing Girls' Menstrual Hygiene Practices in East Africa [3].

The Predesigned Questionnaire was prepared on the material obtained from various previous studies related to the topic. It consisted of two parts - one for filling up the demographic characteristics of students including socio economic status and the other part consisted of questions related to menstrual hygiene practices. The components included: a) Usage of pads b) Bathing during menstruation c) Access to covered toilets f) Duration of normal flow g) Normal interval between the menstrual cycles h) Source of information regarding menstruation and hygiene practices. The questionnaire was prepared in local language. We received 80 answers to the questionnaire.

\section{Result}

All survey items from the questionnaire were translated from research assistants local to the area. This study was carried out in a population of adolescent girls of 22-25 years from Medical faculty. It has revealed the level of knowledge and practices related to menstruation and menstrual hygiene in the study population. It is clear from the study that the level of knowledge about menstruation and menstrual hygiene is adequate because they are medical students.

\section{School attendance}

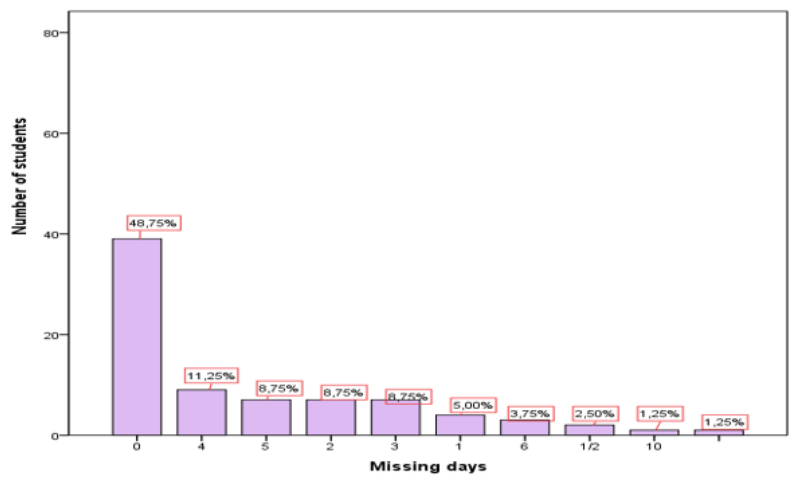

Figure 1: Missing days in one week.

Figure 1 shows that the majority of $48.75 \%$ of students are not absent due to menstrual cramps. $11.25 \%$ were absent about four days a week, while $8.75 \%$ two to 5 days a week. $1.25 \%$ did not answer to this question.

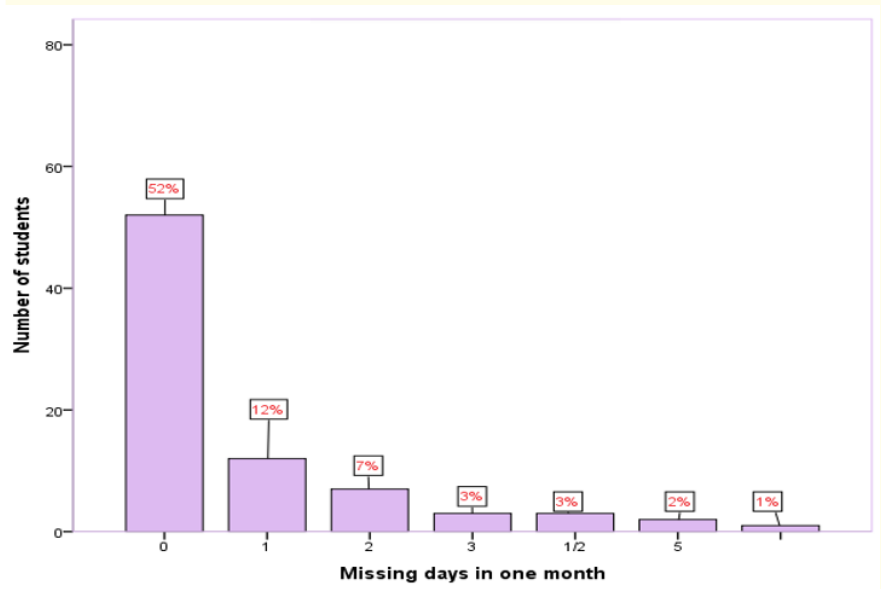

Figure 2: Missing days in one month. 
Figure 2 shows that $52 \%$ of female students are not absent due to menstrual cramps during menstruation, while $16 \%$ were absent from one to five days a month. $1 \%$ of female students did not answer to this question.

\section{Physical activity}

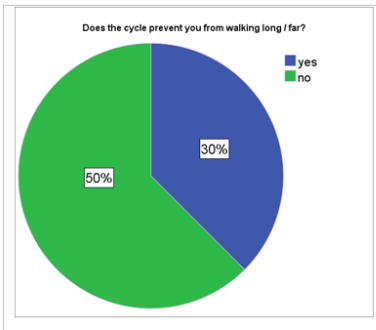

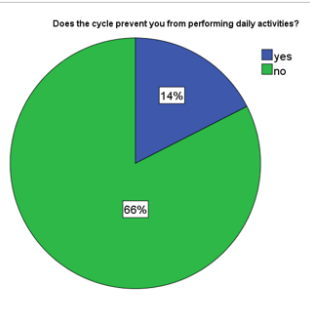

b)
Figure 3: Physical activity and daily activities.

In more than $50 \%$ female students answered that during the menstrual period they are not prevented from doing physical activity, which can be seen in figure 3 a. $66 \%$ of female students gave the answer that the cycle does not prevent them from performing daily activities (Figure 3b).

Pain level

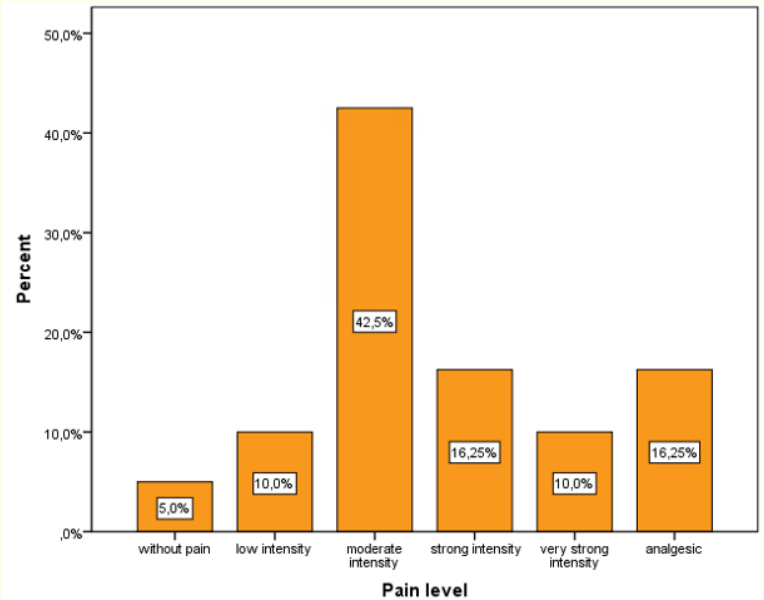

Figure 4: Pain levels that girls have during the menstrual cycle.
Figure 4 shows the percentages of pain levels that girls have during the menstrual cycle. Only $5 \%$ have no pain, $10 \%$ have low intensity, while $70 \%$ have moderate to severe pain, and $16 \%$ should take a painkiller.

Problems

\begin{tabular}{|c|c|c|}
\hline I have nowhere to change & Frequency & Percent \\
\hline Yes & 33 & 41.3 \\
\hline No & 45 & 56.3 \\
\hline
\end{tabular}

Table 1: Conditions for menstrual hygiene management in toilets.

In the section where there are opportunities for remarks, most students stated that they do not have adequate toilets to change, and to remove waste. The table 1 shows a higher percentage of responses in the direction that they have nowhere to change during the menstrual cycle during teaching. Dokovska and Kochubovski in their research noticed that a large number of educational institutions do not have adequate opportunities to maintain menstrual hygiene management [3]. In another cross-sectional study in 3 schools in the Catankudi district of Baticalaloa, there is a shortage of menstrual hygiene practices in adolescent girls, and there must be intensive education to reinforce all components of menstrual hygiene. Strategies such as water access and sanitation can play a major role in adopting safe practices. It was noted that none of the girls in the study had favorable factors necessary for proper menstrual hygiene [4].

Hennegan J in his study says that in order to improve measurement, authors should clearly define the constructs they aim to measure and outline how these were operationalized for measurement. Results indicate the need for the development and validation of new measures, and the evaluation of the performance of existing measures across contexts. In particular, the definition and measurement of menstrual practices, knowledge, attitudes, norms and restrictions should be addressed [5].

\section{Discussion and Conclusions}

This paper is dedicated to the problem of menstrual hygiene in adolescent girls aged 22-25. The problem with a woman's intimate hygiene is important for maintaining her reproductive health. According to the survey results, only $5 \%$ have no pain, $10 \%$ have low intensity, while $70 \%$ have moderate to severe pain, and $16 \%$ 
should take painkillers. So, many adolescent girls had pain during the menstrual cycle. Consultation with a gynecologist at an early period is very important for the future. Girls need to know their rights, but also the health benefits of timely gynecological examinations. Also ultrasound diagnosis of the uterus and ovaries, thyroid gland, consultation with a gynecologist and endocrinologist for girls with severe pain during meniscus, can help solve the problem. It is necessary to qualitatively assess the health of adolescent girls. It is very important for their reproductive function. Adequate menstrual hygiene education and functional, clean and well-equipped WASH facility can also reduce the absence of girls during menstruation. The aim of the study is to find the problems related to menstrual hygiene and to slowly eliminate the shortcomings over time. Research on the approach and knowledge from a timely visit to a gynecologist is ongoing. The education is at the focus. It is aimed 'to increase the life quality' of people by study education. The education of the individuals in the societies, their reach to medical services are quite important. The universities play a significant role in 'lifelong education' of people in the society by cooperating with the other state-private educational institutions. In addition, the universities which have 'Faculty of medicine' and 'research and practice hospitals' fulfil a significant obligation for bringing basic and advanced medical services to the people in the society [6].

Education for girls in relation to menstrual cycles is very important. Based on the obtained results, we can conclude that the awareness of the Medical Students is great, and they have no obstacles in everyday life, this can help us in the further education of the other age groups. The only problem that medical girls have during the menstrual cycle is the place and the appropriate toilets, clean and tidy where they can be changed during the menstrual cycle while they are in college.

Environmental protection and waste management are two notions that complement and complete each other. It is vital for us to understand the nature of our actions and most importantly the consequences derived from our actions. It is very popular nowadays to talk about recycling, and we can even say it is trendy to be an environmentalist. But behind fashion trends and beyond there is a certain need [7].

Recent efforts to better acknowledge pupils' perceptions have helped to provide a more detailed picture on the actual conditions of WASH in schools. A recent survey undertaken by the WHO Re- gional Office for Europe in five countries in the region shows not only the degree of pupil dissatisfaction with school toilets but also the large differences between countries [8].

Adequate education on menstrual hygiene and functional, clean and well-equipped WASH facilities can lower the absenteeism of girls during their period [9].

\section{Bibliography}

1. Guidance on Menstrual Health and Hygiene. UNICEF, March 2019, first edition (2019).

2. Questionnaire Assessing Girls' Menstrual Hygiene Practices in East Africa. Questionnaire, version 11; 31st May (2013).

3. N Dokovska., et al. "Achieving the human right to water and sanitation". UNECE, Geneva (2018): 1-58.

4. Sujirtha N., et al. "Knowledge and Practice of Menstrual Hygiene among Adolescent Girls in Selected Kattankudy area, Batticaloa District". The Journal of Public Health Photon 119 (2017): 255-262.

5. Hennegan J., et al. "Measurement in the study of menstrual health and hygiene: A systematic review and audit". PLOS ONE 15.6 (2020): e0232935.

6. A R Erdem., et al. "Opinions of the Pamukkale University Faculty of Education Students about the Obligation of the University for Social Sustainable Development". Journal of Environmental Protection and Ecology 14 (2013): 1283-1295.

7. D E Popescu., et al. "Waste management strategy at a public". Journal of Environmental Protection and Ecology 17.3 (2016): 1011-1020.

8. V Grossi., et al. "The situation of water, sanitation and hygiene in schools in the Pan-European region". UNECE/WHO, Copenhagen (2016): 1-70.

9. Sumpter $\mathrm{C}$ and Torondel B. "A systematic review of the health and social effects of menstrual hygiene management". PLoS One 8.4 (2013): e62004.

Volume 3 Issue 10 October 2021 (C) All rights are reserved by Aleksandra Petrova., et al. 